\title{
Special Issue On: Optimization for Engineering, Science and Technology
}

Many modern engineering, science and technological problems inevitably faces problems of uncertainty in various aspects such as natural disaster, chaotic decision making, human resource availability, processing capability and constraints and limitations imposed by the authority. This problem has to be solved by a methodology which takes care of such uncertain information. As the analyst solves this problem, the decision maker and the implementer have to coordinate with the analyst for taking up a decision on a successful strategic and holistic decision making for final implementation. Such a complex problems in an imperfect world can be solved by the robust and flexible optimization methodologies.

The objective of the special issue is to enlighten the researchers working on the development of innovative and novel techniques and methodologies to improve the performance of current development on the advanced algorithms related real world practical problems in the research areas of novel and modern optimization and its application in Engineering, Science and Technology.
The special issue includes ten outstanding research papers in the field of optimization and its application in engineering and technology. The editors sincerely thank the referees' and the authors' for their marvelous contributing in making this special issue a successful publication. This special issue will contribute new body of the knowledge to the researchers across the planet.

Sincerely,

Guest Editors

Pandian Vasant*, Universiti Teknologi Petronas,

Malaysia

Junzo Watada, Universiti Teknologi Petronas, Japan

*E-mails: pvasant@gmail.com pandian_m@utp.edu.my 